\title{
Prospective longitudinal assessment of parotid gland function using dynamic quantitative pertechnate scintigraphy and estimation of dose-response relationship of parotid-sparing radiotherapy in head-neck cancers
}

Tejpal Gupta ${ }^{1,2^{*}}$, Chandni Hotwani ${ }^{1}$, Sadhana Kannan², Zubin Master ${ }^{1}$, Venkatesh Rangarajan ${ }^{3}$, Vedang Murthy ${ }^{1}$, Ashwini Budrukkar ${ }^{1}$, Sarbani Ghosh-Laskar ${ }^{1}$ and Jai Prakash Agarwal ${ }^{1}$

\begin{abstract}
Purpose: To estimate dose-response relationship using dynamic quantitative ${ }^{99 m} \mathrm{Tc}$-pertechnate scintigraphy in head-neck cancer patients treated with parotid-sparing conformal radiotherapy.

Methods: Dynamic quantitative pertechnate salivary scintigraphy was performed pre-treatment and subsequently periodically after definitive radiotherapy. Reduction in salivary function following radiotherapy was quantified by salivary excretion fraction (SEF) ratios. Dose-response curves were modeled using standardized methodology to calculate tolerance dose 50 (TD50) for parotid glands.

Results: Salivary gland function was significantly affected by radiotherapy with maximal decrease in SEF ratios at 3 -months, with moderate functional recovery over time. There was significant inverse correlation between SEF ratios and mean parotid doses at 3-months $(r=-0.589, p<0.001)$; 12-months $(r=-0.554, p<0.001)$; 24-months $(r=-0.371$, $p=0.002)$; and 36 -months $(r=-0.350, p=0.005)$ respectively. Using a post-treatment SEF ratio $<45 \%$ as the scintigraphic criteria to define severe salivary toxicity, the estimated TD50 value with its $95 \%$ confidence interval $(95 \% \mathrm{Cl})$ for the parotid gland was 35.1Gy (23.6-42.6Gy), 41.3Gy (34.6-48.8Gy), 55.9Gy (47.4-70.0Gy) and 64.3Gy (55.8-70.0Gy) at 3, 12, 24, and 36 -months respectively.
\end{abstract}

Conclusions: There is consistent decline in parotid function even after conformal radiotherapy with moderate recovery over time. Dynamic quantitative pertechnate scintigraphy is a simple, reproducible, and minimally invasive test of major salivary gland function.

Keywords: Conformal radiotherapy, Dose-response, Salivary scintigraphy, Xerostomia

\footnotetext{
* Correspondence: tejpalgupta@rediffmail.com

${ }^{1}$ Department of Radiation Oncology, Tata Memorial Hospital and ACTREC,

Tata Memorial Centre, Parel, Mumbai 400 012, India

${ }^{2}$ Department of Epidemiology \& Clinical Trials Unit - Clinical Research Secretariat,

Tata Memorial Hospital and ACTREC, Tata Memorial Centre, Parel, Mumbai 400

012, India

Full list of author information is available at the end of the article
} 


\section{Background}

Definitive (chemo) radiotherapy is the contemporary standard of care in the non-surgical management of head and neck squamous cell carcinoma (HNSCC) $[1,2]$. The salivary glands are often incidentally irradiated during comprehensive irradiation of the head-neck cancers resulting in xerostomia that can adversely affect healthrelated quality-of-life (QOL) [3-5]. Xerostomia may result in poor oro-dental hygiene, altered taste sensation, and pain leading to difficulty in speaking, chewing and swallowing [6]. Xerostomia can be defined and graded $[6,7]$ both subjectively according to patient's symptoms (severity of dryness and/or response on stimulation) as well as objectively using quantified saliva production or excretion (salivary flow and/or scintigraphy). Stimulated salivary production is largely derived from the parotid glands while resting or unstimulated saliva is mostly produced by submandibular, sublingual, and various minor salivary glands [8]. Traditionally, salivary gland function has been assessed objectively by flow-rate measurements [9-11]. This can be performed at rest (unstimulated) or in response to administration of a sialogogue (poststimulation). Collection of secretion from each parotid duct orifice via a cannula is the most common method of assessing individual parotid gland function. However, cannulation is an invasive procedure associated with a steep learning curve necessitating technical skill and expertise. It can at times be quite difficult and challenging [11], particularly in the post-treatment setting. Alternatively, whole mouth salivary function can be assessed by asking the patient to produce as much saliva as possible within a given period of time. Such measurementscan be uncertain and variable, with standard deviation of 20$30 \%$ reported for whole-mouth measurements [10]. In recent times, high-precision radiotherapy techniques such as three-dimensional conformal radiotherapy (3D-CRT) and intensity-modulated radiation therapy (IMRT) have gained immense popularity in HNSCC. IMRT produces highly conformal dose distributions with resultant substantial sparing of major salivary glands that can potentially reduce the incidence, duration, and severity of xerostomia with a positive impact on health-related QOL [12-15]. Parotid gland sparing can be further augmented using in-room image-guidance and periodic adaptive replanning during a course of fractionated radiotherapy [16]. With conformal techniques, individual salivary glands may be differentially spared, depending upon their proximity to high-risk areas. Thus, it is important to assess their functional status individually rather than as a whole as is typically assessed by wholemouth measurements. Dynamic salivary gland scintigraphy using ${ }^{99 \mathrm{~m}}$ Tc-pertechnate is a simple, reproducible, and minimally invasive test that provides quantitative estimates of parenchymal and excretory function of individual major salivary glands [17]. It can be a suitable alternative to salivary flow-rate measurements for quantification of post-radiotherapy salivary dysfunction.

\section{Aims}

To report on prospective longitudinal assessment of functional changes in parotid glands using dynamic quantitative ${ }^{99 \mathrm{~m}} \mathrm{Tc}$-pertechnate scintigraphy and estimate their dose-response relationship in a cohort of patients with HNSCC treated using parotid-sparing conformal radiotherapy techniques with or without platinum-based concurrent systemic chemotherapy.

\section{Materials and methods}

Sixty previously untreated patients with early to moderately advanced squamous cell carcinoma of the oropharynx, larynx, or hypopharynx (stage T1-T3, N0-2b) were accrued and treated on an institutional review board approved prospective randomized controlled trial comparing 3D-CRT and IMRT. Suitable patients with locoregionally advanced disease (bulky T2, T3, and/or node positive) also received concurrent chemotherapy. Cisplatin was administered once weekly as an intravenous infusion @30 mg/m ${ }^{2}$ with appropriate hydration, forced saline dieresis, and anti-emetic prophylaxis as per contemporary institutional standard of care. All patients provided written informed consent for participation in this monoinstitutional randomized trial registered at Clinical Trials Registry-India (CTRI/2008/091/000045). Physician-rated acute salivary gland toxicity was the primary endpoint, while patterns of failure, loco-regional control, diseasefree survival, overall survival, QOL, and late xerostomia were secondary endpoints. Details on target volume delineation, treatment planning, and delivery have been published previously $[18,19]$. Salivary gland toxicity (both acute and late xerostomia) was scored subjectively by the treating physicians using Radiation Therapy Oncology Group (RTOG) toxicity criteria [20].

\section{Salivary scintigraphy}

All patients underwent salivary gland scintigraphy using ${ }^{99 \mathrm{~m}}$ Tc-pertechnate prior to initiation of definitive (chemo)radiotherapy. During the initial part of the study, scintigraphy was done using a semi-quantitative methodology, precluding accurate quantification of salivary function. Subsequently, dynamic quantitative ${ }^{99 \mathrm{~m}} \mathrm{Tc}$ pertechnate scintigraphy was performed according to the method described by Klutman [17] in the remaining 41 patients (82 parotid glands) that constitute the dataset of this analysis. Quantitative assessment of the salivary function was performed at baseline (pre-treatment) and subsequently longitudinally at pre-specified time-points on follow-up viz. 3-months $(\mathrm{n}=41)$; 12-months $(\mathrm{n}=38)$; 24months $(\mathrm{n}=35)$; and 36-months $(\mathrm{n}=32)$ after completion 
of definitive (chemo)radiotherapy. Scintigraphy studies were performed with the patient in the supine position under a gamma-camera (Infinia Hawkeye, GE Healthcare, Waukesha, USA) with low-energy high-resolution collimators. No oral stimulus was permitted for 60 minutes before imaging. After intravenous administration of $15 \mathrm{mCi}$ (200 MBq) ${ }^{99} \mathrm{~m}_{\mathrm{Tc}}$-pertechnetate, 30-second sequential frames (anterior view) were acquired and stored in the computer system. Fifteen minutes after injection, salivary stimulation was provided by ingestion of $5 \mathrm{ml}$ of sialogogue (lemon juice). The study was continued for another $10 \mathrm{mi}-$ nutes after sialogogue administration. For analysis of the data, regions of interest were drawn around the right and left parotid and submandibular glands by nuclear medicine physicians and corresponding time-activity curves generated. Background correction was performed using the midline neck region. Time-activity curves were fitted to exponential functions. Salivary excretion fraction (SEF) of an individual salivary gland was quantified by calculating the maximal excretory activity per gland as a fraction of maximal uptake (Figure 1).

\section{Dose-response analysis}

For the dose-response analysis, it was assumed that the glands within an individual patient would not influence each other. Reduction in salivary gland function after (chemo)radiotherapy was described by the relative SEF or SEF ratio defined as the ratio of SEF at time-point $t^{\prime}$ ' after treatment compared to the baseline SEF (pre-treatment) $\mathrm{x} 100 \%$. SEF ratios at different time-points on follow-up (3, 12, 24, and 36-months) were correlated with mean parotid doses. An SEF ratio <45\% [21] was used as an objective scintigraphic criteria to define severe salivary toxicity. This has been shown to correlate best with salivary flow-rate measurements [21] wherein flow-reduction to $<25 \%$ of pre-treatment output is regarded as severe salivary gland toxicity. Dose-response analysis was restricted to individual parotid glands in

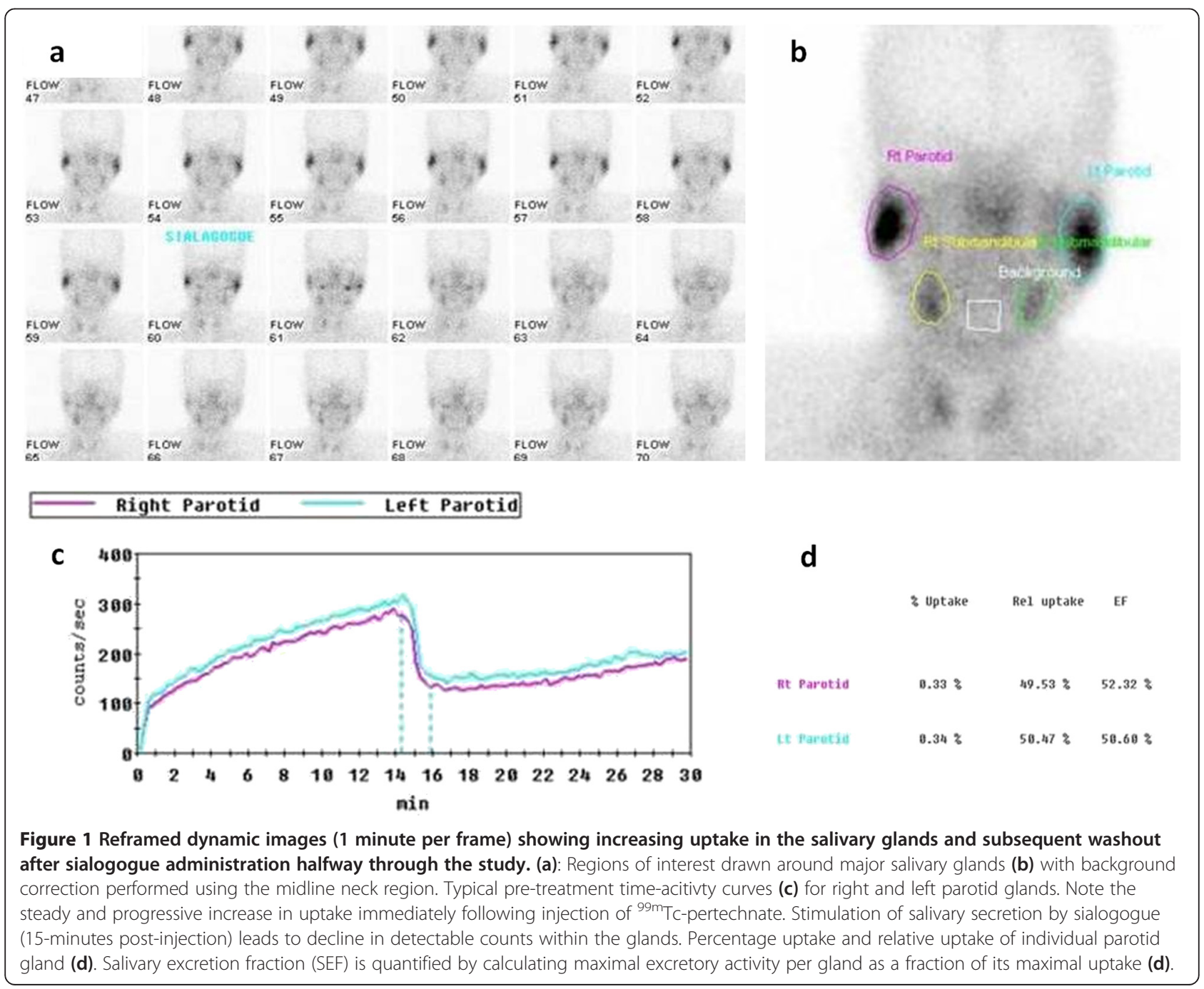


this study. Submandibular glands were not considered for such analyses as they had neither been contoured nor given any dose-volume constraints during radiotherapy planning and optimization. Data were fitted to the Lyman-Kutcher-Burman (LKB) model [22,23] for calculating normal tissue complication probability (NTCP). Briefly, this model assumes that the probability of complications after uniform irradiation of a specified partial volume of an organ follows a sigmoid dose-response relationship. Three parameters in this model are ' $n$ ', ' $m$ ', and tolerance dose 50 (TD50). The parameter ' $\mathrm{n}$ ' accounts for the volume effect of an organ and was considered as 1 for the purpose of this analysis assuming parallel architecture of the parotid glands. The parameter ' $\mathrm{m}$ ' describes the slope of the dose-response curve. The TD50 of partial volume ' $v$ ' is the dose resulting in $50 \%$ probability of a complication for uniform irradiation of that partial volume ' $v$ '. The model requires input of a single parotid gland dose. The multi-step dose-volume histogram (DVH) was transformed to a single-step DVH with an effective partial volume irradiated uniformly by a reference dose. The inputs to the model were transformed DVH and parotid gland function that was adjusted as a binary response variable on the basis of each individual gland. A maximum likelihood method was used to fit the model to the complication data and find the best estimate and 95\% confidence intervals (95\% CI) for the model parameters. In an exploratory analyses, dose-response curves were also generated to estimate TD50 values and the corresponding slope $(\mathrm{m})$ using different SEF ratios to define severe salivary gland toxicity (ranging from SEF ratio $<75 \%$ to $<25 \%$ ). Agreement between subjective xerostomia scores (RTOG grading) and objective scintigraphic criteria (SEF ratio $<45 \%$ ) was tested using the kappa statistic.

\section{Results}

Relevant demographic, clinical, and dosimetric characteristics of the study cohort $(n=41)$ are described in Table 1. The mean and standard deviation (SD) of mean doses to ipsilateral and contralateral parotid glands were 48.3Gy (13.0) and 39.7Gy (12.8) respectively. With IMRT, the median and its inter-quartile range (IQR) of mean doses to the ipsilateral parotid gland was 37.2Gy (30.449.0Gy) compared to 59.3Gy (51.2-63.8Gy) with 3D-CRT $(\mathrm{p}<0.001)$. The contralateral parotid gland was also spared significantly with IMRT. The median of mean doses with its IQR to the contralateral parotid gland was 28.1Gy (25.2-30.4Gy) with IMRT which was significantly lesser than 53.3Gy (43.8-56.4Gy) with 3D-CRT ( $<$ 0.0001).

The pre-treatment (baseline) SEF was normally distributed for both parotid glands with a mean value of $50.1 \%(\mathrm{SD}=14.1)$. However, considerable variability of parotid gland output was noted with baseline SEFs
Table 1 Demographic and treatment characteristics of study cohort $(\mathbf{N}=\mathbf{4 1})$

\begin{tabular}{ll}
\hline Characteristics & Number \\
\hline Age: & \\
\hline Median & 54 years \\
Range & $33-65$ years \\
Gender: & \\
\hline Male & $37(90.2 \%)$ \\
Female & $04(09.8 \%)$ \\
Primary site: & \\
\hline Oropharynx & $23(56.1 \%)$ \\
Larynx & $10(24.4 \%)$ \\
Hypopharynx & $08(19.5 \%)$ \\
Laterality (epicentre): & \\
\hline Right & $22(53.7 \%)$ \\
Left & $18(43.9 \%)$ \\
Midline & $01(02.4 \%)$ \\
American Joint Committee on Cancer (AJCC) staging: & \\
\hline Stage II & $09(21.9 \%)$ \\
Stage III & $17(41.5 \%)$ \\
Stage IV & $15(36.6 \%)$ \\
Radiotherapy technique: & \\
\hline Three-Dimensional Conformal Radiotherapy (3D-CRT) & $20(48.8 \%)$ \\
Intensity-Modulated Radiation Therapy (IMRT) & $21(51.2 \%)$ \\
Median (inter-quartile range) of mean parotid dose: & \\
\hline Ipsilateral parotid & $30.0 \mathrm{~Gy}(36.2-59.7)$ \\
Contralateral parotid & $35.4 \mathrm{~Gy}(28.0-53.5)$ \\
\hline Yes & \\
No & \\
\hline
\end{tabular}

ranging from $10-70 \%$. No significant pre-treatment differences were found between the right and left parotid gland SEFs. Baseline scintigraphic parameters were not dependent on age, gender, or stage. The parenchymal as well as the excretory function of all major salivary glands was significantly affected by (chemo)radiotherapy with resultant decrease in SEF ratios at 3-months following completion of therapy. At 12-months post-treatment, there was modest functional recovery of the parotid glands (contralateral >>ipsilateral), which improved progressively further till 24-months, but reached a plateau somewhat thereafter. The median SEF ratios (IQR) of the parotid glands were $25.7 \%(0-55.8 \%), 38.2 \%$ (3.8-68.9\%), 59.0\% (8.4-83.6\%), and 65.3\% (29.4-95.4\%) at 3-months, 12-months, 24-months and 36-months respectively (Figure 2) after (chemo)radiotherapy indicating substantial recovery of salivary function over time, mostly within the first two years on follow-up. 


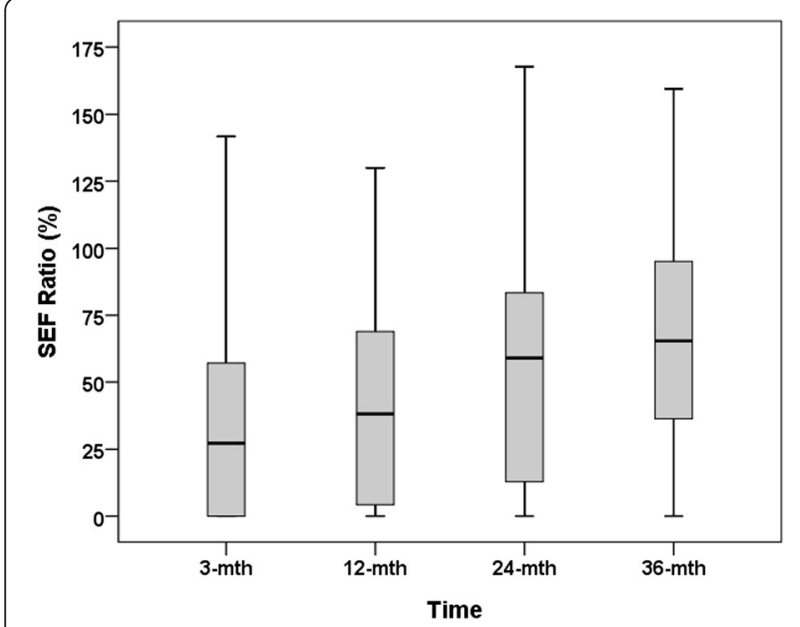

Figure 2 Boxplot showing median salivary excretion fraction (SEF) ratios at 3-months, 12-months, 24-months, and 36-months after (chemo) radiotherapy. Note the moderate recovery of salivary function continuing till 2-years post-treatment.

There was significant inverse correlation (Figures 3a-d) between SEF ratios and mean parotid doses at 3-months $(\mathrm{r}=-0.589, \mathrm{p}<0.001)$; 12-months $(\mathrm{r}=-0.554, \mathrm{p}<0.001)$; 24-months $(\mathrm{r}=-0.371, \mathrm{p}=0.002)$; and 36-months $(\mathrm{r}=-0.350, \mathrm{p}=0.005)$ respectively (see online Additional file 1: Table S1). Using a post-treatment SEF ratio $<45 \%$ as the scintigraphic criteria to define severe salivary gland toxicity [21], the estimated TD50 (95\% CI) values for the parotid glands at 3-months, 12-months, 24-months, and 36-months were 35.1Gy (23.6-42.6Gy), 41.3Gy (34.648.8Gy), 55.9Gy (47.4-70.0Gy) and 64.3Gy (55.8-70.0Gy) respectively (Figure 4a-d). The upper limits of the 95\% CI of TD50 estimates at 24 and 36 months could not be computed accurately, as the dose-response curves lost some of their sigmoidal nature and became somewhat flatter over time. The corresponding ' $m$ values (slope of the doseresponse curve) were $0.48,0.38,0.44,0.38$ for the four time-points respectively. Using the Quantitative Analysis of Normal Tissue Effects in the Clinic (QUANTEC) 20/20 rule [7], the incidence of severe toxicity $(\mathrm{SEF}<45 \%)$ was estimated at $23 \%$ at 3-months, but decreased to $13 \%$ and $9 \%$ at 12-months and 24-months respectively, providing external validation of the QUANTEC guidelines in predicting a low probability of severe xerostomia. There was poor to weak agreement between subjective scores (physicianrated RTOG salivary gland toxicity) and objective scintigraphic criteria (toxicity defined as SEF ratio $<45 \%$ ) at all four post-treatment time-points (see online Additional file 2: Table S2). Results of the exploratory analyses estimating the TD50 values and the slope $(\mathrm{m})$ of the doseresponse curve at all four time-points using different SEF ratios to define severe salivary gland toxicity are also summarized (see online Additional file 3: Table S3).

\section{Discussion}

Curative-intent radiotherapy for head-neck cancers often leads to irreversible impairment of salivary function and consequent xerostomia that adversely affects healthrelated QOL [3-5]. This decline in salivary function occurs even after parotid-sparing conformal radiotherapy albeit to a lesser degree (both in terms of incidence and severity) particularly with IMRT [19] with substantial recovery over time. The use of IMRT in clinical practice has resulted in improved tolerance to treatment [24] for patients with head-neck cancer and reduced delayed or late effects. Dose-response relationship for major salivary glands has traditionally been based on salivary flow-rate measurements [9-11]. In particular, a strong correlation has been shown between the mean parotid dose and residual post-radiotherapy salivary function [7]. There is a gradual decrease in salivary flow with increasing mean parotid dose. Minimal functional impairment occurs at mean doses $<10-15 \mathrm{~Gy}$, increasing doses (in the range of 20-30Gy) leads to progressive deterioration with severe xerostomia occurring at mean parotid doses of $>40 \mathrm{~Gy}$. The TD50 for the endpoint of severe xerostomia (traditionally defined as reduction in salivary flow rate to $<25 \%$ of pre-treatment value) has been quite variable with estimates ranging from 20-45 Gy [7].

Eisbruch et al. [9] described a steep dose-response relationship for the parotid glands in a cohort of 88 patients treated with IMRT with estimated 1-year TD50 of 28 Gy using salivary flow-rate measurements. Using similar methodology, Chao et al. [10] also reported a TD50 of $32 \mathrm{~Gy}$. In contrast, Roesink et al. [11] found no threshold dose in 108 patients of head-neck cancer treated with conventional techniques but reported a TD50 of 39 Gy at 1-year using flow-data. The largest dataset of parotid gland function measurements at 1-year (combining the Michigan and Utrecht experience) reported a TD50 of 39.9 Gy and a complication probability of $17-26 \%$ with mean parotid doses in the range of 25-30 Gy [25].

While flow-rate measurements have remained the benchmark for assessment of salivary function, given their limitations, dynamic quantitative pertechnate scintigraphy has emerged as a simple, reproducible, and minimally invasive test for quantification of postradiotherapy salivary function of individual major salivary glands. Unlike, salivary flow-rate measurements, there has been a lack of consensus on the definition of severe salivary toxicity using scintigraphic criteria. In the largest scintigraphic dataset $(n=96)$, Roesink et al. [21] reported significant correlation between SEF ratios and mean parotid doses, both in early (6-weeks) and later (1-year) follow-up. They also modeled the doseresponse curves using different SEF ratios to define severe salivary toxicity due to relative lack of previous 

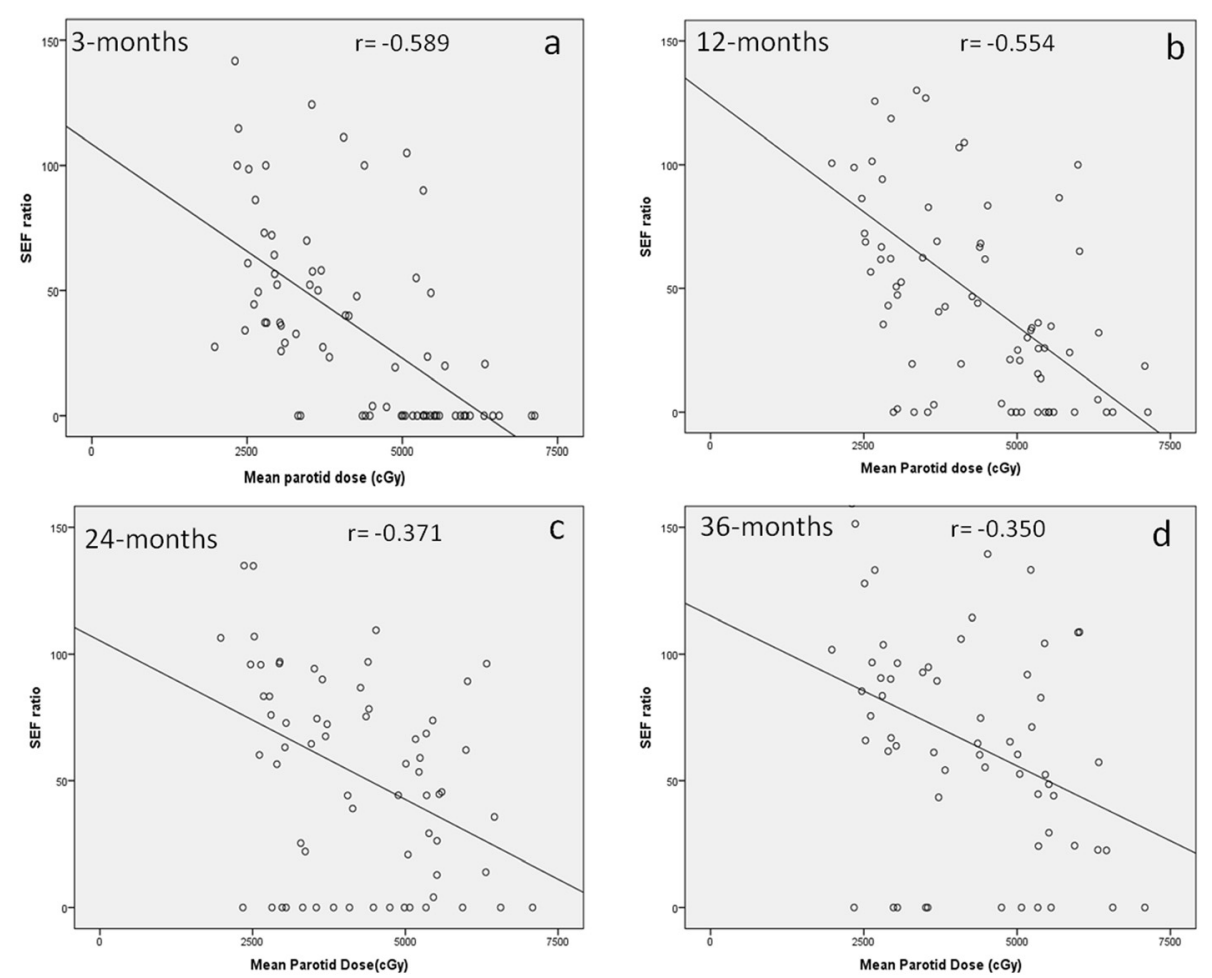

Figure 3 Salivary excretion fraction (SEF) ratio as a function of mean parotid dose at 3-months (a), 12-months (b), 24-months (c), and 36-months (d) respectively. Note the significant inverse correlation between the two at all time-points.

reports fitting scintigraphic data to NTCP models. Severe salivary toxicity defined as SEF ratio $<45 \%$ gave TD50 estimates that were comparable to their flow-data at 6-weeks and 1-year after treatment. The Heidelberg group has consistently used SEF ratio $<50 \%$ to define severe salivary toxicity and TD50 $(95 \% \mathrm{CI})$ estimates for the parotid gland of about 35Gy (95\% CI = 20-45Gy) between 2-6 months post-treatment [26-28]. Recent times have witnessed more widespread use of salivary scintigraphy for postradiotherapy assessment of salivary dysfunction. The estimated tolerance doses for the parotid gland in selected studies [21,26-31] using quantitative salivary scintigraphy are summarized in Table 2 . The reported variability in scintigraphy-based TD50 values is somewhat lesser compared to flow-based estimates. Also scintigraphy-based TD50 estimates have generally tended to be higher than their flow-based counterparts. At this point, salivary scintigraphy cannot be considered superior to salivary flow-rate measurements, but can be a viable practical alternative. The reported variation in reported TD50 values (both for salivary flow data as well as scintigraphic data) could be a result of differences in radiotherapy techniques and resultant dose distributions, fraction-size effects, intra-gland sensitivity, use of concurrent chemotherapy, methods of measurement, definition of toxicity, timepoints of assessment, and NTCP models used for such calculation. Semenenko and $\mathrm{Li}$ [32] in a pooled analysis of published clinical data to provide population LKB-NTCP model parameters for incorporation in treatment planning estimated a TD50 (95\% CI) of 31.4Gy (29.1-34.0Gy) for the endpoint of reduction in stimulated salivary flow below $25 \%$ within six months after radiotherapy.

\section{Strengths and limitations}

The TD50 (95\% CI) estimates for the parotid glands in this study were derived from a prospective cohort of patients. Hence they do not suffer from inherent limitations of any retrospective analyses. The relatively wide dispersion of mean parotid doses in the study allowed for more robust curve fitting at both ends of the spectrum. Serial follow-up provided an opportunity to estimate longitudinal recovery of salivary function over time and calculate TD50 values at longer follow-up times (2 and 3-years) than is generally reported in the literature (typically up to 1-year). However, the doseresponse curves became somewhat flatter over time precluding accurate computation of the upper limits of the $95 \%$ CIs of the TD50 estimates at 24 and 36-months. Dose-response analyses was restricted to parotid glands only in the study thereby precluding such modeling for submandibular salivary glands which are the major contributors to salivation in the resting state. Intentional 

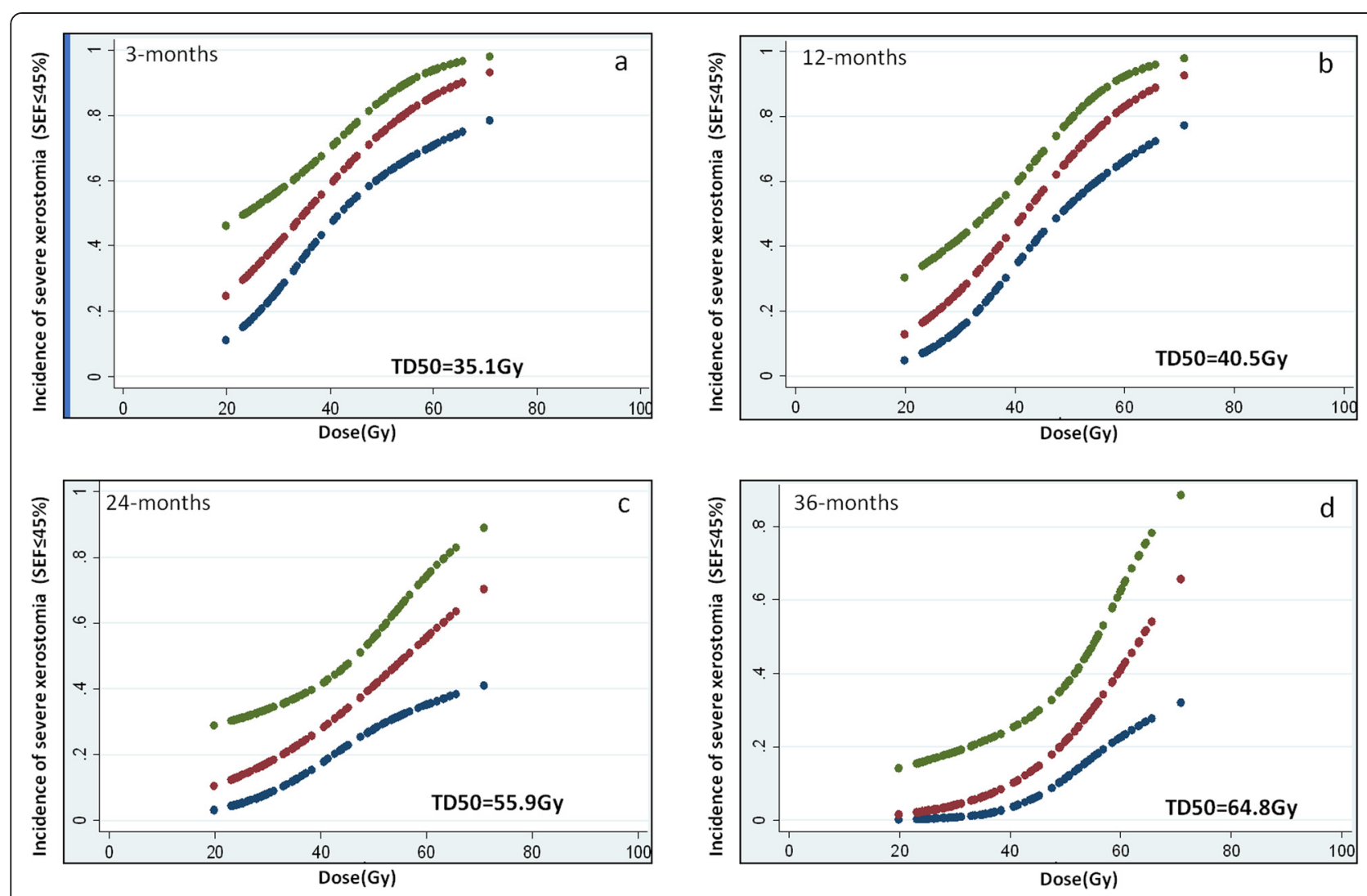

Figure 4 Fitted dose-response curves for normal tissue complication probability of severe xerostomia (defined as SEF ratio $<45 \%$ ) as a function of mean parotid dose at 3-months (a), 12-months (b), 24-months (c), and 36-months (d) respectively. Upper and lower curves represent $95 \%$ confidence intervals for the fitted model.

Table 2 Studies estimating tolerance dose 50 (TD50) of parotid glands using salivary scintigraphy

\begin{tabular}{|c|c|c|c|c|c|}
\hline \multirow[t]{2}{*}{ Study (ref) } & \multirow{2}{*}{$\begin{array}{l}\text { Number of } \\
\text { patients }(N)\end{array}$} & \multirow{2}{*}{$\begin{array}{l}\text { Mean parotid } \\
\text { dose }\end{array}$} & \multirow{2}{*}{$\begin{array}{l}\text { Salivary scintigraphy criteria for } \\
\text { defining severe xerostomia }\end{array}$} & \multicolumn{2}{|c|}{ Tolerance Dose $50(95 \% \mathrm{Cl})$} \\
\hline & & & & 6 weeks- 6 months & 1-year \\
\hline \#Roesink [21] & 96 (conv) & 33.14Gy & SEF ratio $<45 \%$ & 29Gy (25-34Gy) & 43Gy (37-51Gy) \\
\hline Munter [26] & 18 (IMRT) & NR & SEF ratio $<50 \%$ & 34.8Gy (27.6-42Gy) & NR \\
\hline \multirow[t]{2}{*}{ Munter [27] } & 33 (conv) & 60.6Gy & SEF ratio $<50 \%$ & 36.4Gy (20.5-42.3Gy) & NR \\
\hline & 19 (IMRT) & 27.7Gy & SEF ratio $<50 \%$ & 35Gy (28-42Gy) & \\
\hline \multirow[t]{2}{*}{ *Rudat [28] } & 34 (conv) & $60.7 \mathrm{~Gy}$ & SEF ratio $<50 \%$ & NR & 51.1Gy (43.5-58.7Gy) \\
\hline & 31 (IMRT) & 30.9Gy & & & \\
\hline Tenhunen [29] & 20 (IMRT) & $27.6 \mathrm{~Gy}$ & SEF ratio $<50 \%$ & 40.3Gy (30-53.6Gy) & 39.2Gy (27.9-50.2Gy) \\
\hline Kapanen [30] & 25 (IMRT) & 23.2Gy & SEF ratio $<50 \%$ & 30.4Gy (23.2-37.6Gy) & NR \\
\hline \multirow[t]{2}{*}{ Chen [31] } & 31 (IMRT) & 51.7Gy IL & SEF ratio $<45 \%$ & NR & 43.6Gy (41.3-45.9Gy) \\
\hline & & 36.7Gy CL & & & \\
\hline \multirow[t]{2}{*}{ Present study } & 41 (3D-CRT and IMRT) & 48.3Gy IL & SEF ratio $<45 \%$ & 35.1Gy (23.6-42.6Gy) & 41.3Gy (34.6-48.8) \\
\hline & & 39.7Gy CL & & & \\
\hline
\end{tabular}

$\mathrm{Cl}=$ confidence interval; $\mathrm{SEF}=$ salivary excretion fraction; conv = conventional; 3D-CRT = three dimensional conformal radiotherapy; IMRT = intensity modulated radiation therapy; $\mathrm{NR}=$ not reported; $\mathrm{IL}=$ ipsilateral; $\mathrm{CL}=$ contralateral.

\#First report correlating salivary flow measurements with scintigraphic dataset; SEF ratio $<45 \%$ best correlated with flow data becoming the benchmark scintigraphic criteria defining severe xerostomia.

*Updated results from previous publication (ref) reporting delayed xerostomia; conventional radiotherapy plus amifostine group has been excluded from these estimates. 
sparing of contralateral submandibular salivary gland leads to better preservation of salivary function without any increased risk of marginal failure in the vicinity of the spared gland [33]. Vast majority of patients in the study also received concurrent weekly cisplatin that could possibly influence salivary toxicity. Although cisplatin alone per se does not cause significant salivary dysfunction, its use as a sensitizer concurrently with radiotherapy increases biologically delivered doses potentially enhancing radiotherapy-induced salivary gland toxicity. In addition to physician-rated xerostomia, salivary scintigraphy was used as an objective test to quantify postradiotherapy salivary dysfunction. However, this study did not use salivary-flow measurements generally considered the benchmark for such quantification. Lack of consensus criteria for defining severe salivary toxicity using scintigraphy was another limitation of the study. Nevertheless, various SEF ratio cut-offs were used to define salivary toxicity in an exploratory analyses, although SEF $<45 \%$ was used in the final analysis, interpretation, and reporting. What is also reassuring is that the TD50 estimates (particularly at 1-year) obtained in this study are pretty similar to previously published data of salivary scintigraphy. Although, patients filled QOL forms at baseline and serially longitudinally on follow-up, a xerostomia-specific questionnaire was not used in this study to assess patient-reported outcomes (self-rated xerostomia). Finally, there was poor to weak agreement between subjective xerostomia scores and objective scintigraphic criteria suggesting that observer-based monitoring may underestimate actual xerostomia mandating the need for patient-reported outcomes for such estimation. Large variability in salivary gland function between patients, poor correlation between objective and subjective assessment of salivary toxicity, and limitations of statistical modeling make accurate prediction of salivary dysfunction in an individual patient difficult and challenging.

The tolerance dose estimates for different measures used to describe high-grade xerostomia viz. salivary flow-rates, observer-rated subjective xerostomia, and patient-reported QOL outcomes on a xerostomia-specific questionnaire can be very different. Miah and colleagues [34] reported increasing TD50 values from parotid flow-rates (23.4Gy), subjective xerostomia (33.3Gy), RTOG-graded subjective xerostomia (42.9Gy), and patientreported QOL outcomes (51.6Gy). In the largest analysis $(\mathrm{n}=237$ patients) using patient-reported QOL data of moderate to severe xerostomia [35], the fitted doseresponse curves (LKB-NTCP model) yielded a TD50 of 37.8Gy and 43.9Gy at 3-months and 1-year respectively. Reassuringly, another study [36] that used patient-reported QOL outcomes for fitting to the dose-response curve for NTCP of incidence of $\geq$ grade 3 xerostomia reported a
TD50 of 44.1Gy for the parotid glands 1-year after radiotherapy which was very similar to their TD50 value of 43.6Gy [31,36] estimated using salivary scintigraphy (SEF ratio $<45 \%)$.

\section{Conclusions}

Xerostomia remains an important toxicity following curative-intent irradiation of head-neck cancers. There is consistent and significant decline in parotid gland function even after conformal radiotherapy, albeit to a lesser degree, particularly with IMRT, compared to conventional radiotherapy. However, parotid gland function recovers moderately on longer follow-up, as evidenced by progressively higher SEF ratios and TD50 values over time. Dynamic ${ }^{99 \mathrm{~m}}$ Tc-pertechnate scintigraphy is a simple, reproducible, and minimally invasive test of major salivary gland function that may be a suitable alternative to salivary flow-rate measurements in clinical practice for quantification of postradiotherapy salivary dysfunction.

\section{Additional files}

Additional file 1: Correlation of SEF ratio with mean parotid dose at various time-points on follow-up.

Additional file 2: Test of agreement between subjective xerostomia scores and objective scintigraphic criteria.

Additional file 3: Estimated tolerance dose 50 (TD50) in Gy and corresponding slope $(\mathrm{m})$ of the dose-response curve for the parotid gland at different time-points on serial follow-up using different SEF ratios to define severe salivary toxicity.

\section{Competing interests}

The authors declare that they have no competing interests.

\section{Authors' contributions}

The index randomized controlled trial comparing 3D-CRT and IMRT as well as this study report was conceptualized, designed, conducted, interpreted, and reported by TG. $\mathrm{CH}$ helped with data collection and wrote the first draft of the manuscript. SK and ZM did scintigraphy data analysis and statistical curve fitting. VR interpreted scintigraphic data and helped revise the manuscript. $V M$ and $A B$ helped with literature review and manuscript preparation. SGL and JP were involved in study design, conduct, and critical review of the manuscript. All authors read and approved the final manuscript.

\section{Acknowledgements}

The index randomized controlled trial comparing 3D-CRT and IMRT was partially funded by a research grant from Siemens Oncology Care Systems, USA. The sponsor had no role in study design, conduct, data collection, analyses, interpretation, or reporting (manuscript writing and decision to submit for publication). No financial support was involved in the preparation of this manuscript.

Presentation: Presented in part in the best paper award session at the Indian Cancer Congress (ICC) - 2013, New Delhi, INDIA.

\section{Author details}

'Department of Radiation Oncology, Tata Memorial Hospital and ACTREC, Tata Memorial Centre, Parel, Mumbai 400 012, India. ²Department of Epidemiology \& Clinical Trials Unit - Clinical Research Secretariat, Tata Memorial Hospital and ACTREC, Tata Memorial Centre, Parel, Mumbai 400 012, India. ${ }^{3}$ Department of Nuclear Medicine \& Molecular Imaging, Tata Memorial Hospital and ACTREC, Tata Memorial Centre, Parel, Mumbai 400 012, India. 
Received: 30 October 2014 Accepted: 26 February 2015

\section{Published online: 15 March 2015}

\section{References}

1. Pignon JP, Bourhis J, Domenge C, Designe L. Chemotherapy added to locoregional treatment for head and neck squamous-cell carcinoma: three meta-analyses of updated individual data. MACH-NC Collaborative Group. Meta-Analysis of Chemotherapy on Head and Neck Cancer. Lancet. 2000;355:949-55.

2. Pignon JP, le Maitre A, Maillard E, Bourhis J, on behalf of the MACH-NC Collaborative Group. Meta-analysis of chemotherapy in head and neck cancer (MACH-NC): an update on 93 randomised trials and 17,346 patients. Radiother Oncol. 2009;92:4-14.

3. Dirix P, Nuyts S, Van den Bogaert W. Radiation-induced xerostomia in patients with head and neck cancer: a literature review. Cancer. 2006;107:2525-34.

4. Braam PM, Roesink JM, Raaijmakers CP, Busschers WB, Terhaard CH. Quality of life and salivary output in patients with head-and-neck cancer five years after radiotherapy. Radiat Oncol. 2007;2:3.

5. Ramaekers BL, Joore MA, Grutters JP, van den Ende P, Jong J, Houben R, et al. The impact of late treatment-toxicity on generic health-related quality of life in head and neck cancer patients after radiotherapy. Oral Oncol. 2011:47:768-74.

6. Jensen SB, Pedersen AM, Vissink A, Andersen E, Brown CG, Davies AN, et al. A systematic review of salivary gland hypofunction and xerostomia induced by cancer therapies: prevalence, severity and impact on quality of life. Support Care Cancer. 2010;18:1039-60.

7. Deasy JO, Moiseenko V, Marks L, Chao KS, Nam J, Eisbruch A. Radiotherapy dose-volume effects on salivary gland function. Int J Radiat Oncol Biol Phys. 2010;76:S58-63.

8. Dawes C, Wood CM. The contribution of oral minor mucous gland secretions to the volume of whole saliva in man. Arch Oral Biol. 1973;18:337-42.

9. Eisbruch A, Ten Haken RK, Kim HM, Marsh LH, Ship JA. Dose, volume, and function relationships in parotid salivary glands following conformal and intensity-modulated irradiation of head and neck cancer. Int J Radiat Oncol Biol Phys. 1999;45:577-87.

10. Chao KS, Deasy JO, Markman J, Haynie J, Perez CA, Purdy JA, et al. A prospective study of salivary function sparing in patients with head-andneck cancers receiving intensity-modulated or three-dimensional radiation therapy: initial results. Int J Radiat Oncol Biol Phys. 2001;49:907-16.

11. Roesink JM, Moerland MA, Battermann JJ, Hordijk GJ, Terhaard CH. Quantitative dose-volume response analysis of changes in parotid gland function after radiotherapy in the head-and-neck region. Int J Radiat Oncol Biol Phys. 2001:51:938-46.

12. van Rij CM, Oughlane-Heemsbergen WD, Ackerstaff AH, Lamers EA, Balm AJ, Rasch CR. Parotid gland sparing IMRT for head and neck cancer improves xerostomia related quality of life. Radiat Oncol. 2008;3:41.

13. Vergeer MR, Doornaert PA, Rietveld DH, Leemans CR, Slotman BJ, Langendijk JA. Intensity-modulated radiotherapy reduces radiation-induced morbidity and improves health-related quality of life: results of a nonrandomized prospective study using a standardized follow-up program. Int J Radiat Oncol Biol Phys. 2009;74:1-8.

14. Rathod S, Gupta T, Ghosh-Laskar S, Murthy V, Budrukkar A, Agarwal J. Quality-of-life (QOL) outcomes in patients with head and neck squamous cell carcinoma (HNSCC) treated with intensity-modulated radiation therapy (IMRT) compared to three-dimensional conformal radiotherapy (3D-CRT): evidence from a prospective randomized study. Oral Oncol. 2013:49:634-42.

15. Scott-Brown M, Miah A, Harrington K, Nutting C. Evidence-based review: quality of life following head and neck intensity-modulated radiotherapy. Radiother Oncol. 2010;97:249-57.

16. Castelli J, Simon A, Louvel G, Henry O, Chajon E, Nassef M, et al. Impact of head and neck cancer adaptive radiotherapy to spare the parotid glands and decrease the risk of xerostomia. Radiat Oncol. 2015;10:6.

17. Klutmann S, Bohuslavizki KH, Kroger S, Bleckmann C, Brenner W, Mester J, et al. Quantitative salivary gland scintigraphy. J Nucl Med Technol. 1999;27:20-6.

18. Murthy V, Gupta T, Kadam A, Ghosh-Laskar S, Budrukkar A, Phurailatpam R, et al. Time trial: a prospective comparative study of the time-resource burden for three-dimensional conformal radiotherapy and intensity-modulated radiotherapy in head and neck cancers. J Cancer Res Ther. 2009;5:107-12.

19. Gupta T, Agarwal J, Jain S, Phurailatpam R, Kannan S, Ghosh-Laskar S, et al. Three-dimensional conformal radiotherapy (3D-CRT) versus intensity modulated radiation therapy (IMRT) in squamous cell carcinoma of the head and neck: a randomized controlled trial. Radiother Oncol. 2012;104:343-8.

20. Cox JD, Stetz J, Pajak TF. Toxicity criteria of the Radiation Therapy Oncology Group (RTOG) and the European Organization for Research and Treatment of Cancer (EORTC). Int J Radiat Oncol Biol Phys. 1995;31:1341-6.

21. Roesink JM, Moerland MA, Hoekstra A, Van Rijk PP, Terhaard CH. Scintigraphic assessment of early and late parotid gland function after radiotherapy for head-and-neck cancer: a prospective study of dose-volume response relationships. Int J Radiat Oncol Biol Phys. 2004;58:1451-60.

22. Lyman JT. Complication probability as assessed from dose-volume histograms. Radiat Res Suppl. 1985;8:S13-9.

23. Deasy JO. Comments on the use of the Lyman-Kutcher-Burman model to describe tissue response to nonuniform irradiation. Int J Radiat Oncol Biol Phys. 2000;47:1458-60.

24. Studer G, Linsenmeier C, Riesterer O, Najafi Y, Brown M, Yousefi B, et al. Late term tolerance in head neck cancer patients irradiated in the IMRT era. Radiat Oncol. 2013:8:259.

25. Dijkema T, Raaijmakers CP, Ten Haken RK, Roesink JM, Braam PM, Houweling $A C$, et al. Parotid gland function after radiotherapy: the combined michigan and utrecht experience. Int J Radiat Oncol Biol Phys. 2010;78:449-53.

26. Munter MW, Karger CP, Hoffner SG, Hof H, Thilmann C, Rudat V, et al. Evaluation of salivary gland function after treatment of head-and-neck tumors with intensity-modulated radiotherapy by quantitative pertechnetate scintigraphy. Int J Radiat Oncol Biol Phys. 2004;58:175-84.

27. Munter MW, Hoffner S, Hof H, Herfarth KK, Haberkorn U, Rudat V, et al. Changes in salivary gland function after radiotherapy of head and neck tumors measured by quantitative pertechnetate scintigraphy: comparison of intensity-modulated radiotherapy and conventional radiation therapy with and without Amifostine. Int J Radiat Oncol Biol Phys. 2007;67:651-9.

28. Rudat V, Munter M, Rades D, Grotes KA, Bajrovic A, Haberkorn U, et al. The effect of amifostine or IMRT to preserve the parotid function after radiotherapy of the head and neck region measured by quantitative salivary gland scintigraphy. Radiother Oncol. 2008;89:71-80.

29. Tenhunen M, Collan J, Kouri M, Kangasmaki A, Heikkonen J, Kairemo K, et al. Scintigraphy in prediction of the salivary gland function after gland-sparing intensity modulated radiation therapy for head and neck cancer. Radiother Oncol. 2008;87:260-7.

30. Kapanen M, Collan J, Saarilahti K, Heikkonen J, Kairemo K, Tenhunen M, et al. Accuracy requirements for head and neck intensity-modulated radiation therapy based on observed dose response of the major salivary glands. Radiother Oncol. 2009:93:109-14.

31. Chen WC, Lai CH, Lee TF, Hung CH, Liu KC, Tsai MF, et al. Scintigraphic assessment of salivary function after intensity-modulated radiotherapy for head and neck cancer: correlations with parotid dose and quality of life. Oral Oncol. 2013:49:42-8.

32. Semenenko VA, Li XA. Lyman-Kutcher-Burman NTCP model parameters for radiation pneumonitis and xerostomia based on combined analysis of published clinical data. Phys Med Biol. 2008;53:737-55.

33. Gensheimer MF, Liao JJ, Garden AS, Laramore GE, Parvathaneni U, et al. Submandibular gland-sparing radiation therapy for locally advanced oropharyngeal squamous cell carcinoma: patterns of failure and xerostomia outcomes. Radiat Oncol. 2014:9:255.

34. Miah AB, Gulliford SL, Clark CH, Bhide SA, Zaidi SH, Newbold KL, et al. Dose-response analysis of parotid gland function: what is the best measure of xerostomia? Radiother Oncol. 2013;106:341-5.

35. Lee TF, Fang FM. Quantitative analysis of normal tissue effects in the clinic (QUANTEC) guideline validation using quality of life questionnaire datasets for parotid gland constraints to avoid causing xerostomia during head-and-neck radiotherapy. Radiother Oncol. 2013;106:352-8.

36. Lee TF, Chao PJ, Wang HY, Hsu HC, Chang P, Chen WC, et al. Normal tissue complication probability model parameter estimation for xerostomia in head and neck cancer patients based on scintigraphy and quality of life assessments. BMC Cancer. 2012:12:567. 